\title{
Assessment of ventilation-perfusion mismatching in mechanically ventilated patients
}

\author{
M. Ferrer*, E. Zavala+, O. Díaz*, J. Roca*, P.D. Wagner\#, R. Rodriguez-Roisin*
}

\begin{abstract}
Assessment of ventilation-perfusion mismatching in mechanically ventilated patients. $M$. Ferrer, E. Zavala, O. Díaz, J. Roca, P.D. Wagner, R. Rodriguez-Roisin. OERS Journals Ltd 1998.

ABSTRACT: The multiple inert gas elimination technique (MIGET) is a robust tool to assess both ventilation-perfusion $\left(V^{\prime} \mathrm{A} / Q^{\prime}\right)$ distributions and the role of extrapulmonary factors determining arterial oxygenation during spontaneous breathing and in mechanically ventilated patients. Mixed expired gas sampling used in the MIGET is most often obtained from a 10-L mixing box (10L-MB) placed in the expiratory side of the ventilator circuit. Consequently, a considerable increase in the compression volume $(V c)$ would be expected which, in turn, can give rise to potential errors in the estimation of the effective tidal volume delivered to the patient.
\end{abstract}

The effects of the 10L-MB on the $V_{c}$ were compared with those produced by a newly designed 1-L mixing box (1L-MB).

At a given peak pressure ( $P$ peak) within the ventilator circuit, the $V_{c}$ generated by the 10L-MB was about six-times higher than that produced by the $1 \mathrm{~L}-\mathrm{MB}$. At a $P$ peak $=50 \mathrm{cmH}_{2} \mathrm{O}$, the $V_{\mathrm{c}}$ were $377 \mathrm{~mL}(10 \mathrm{~L}-\mathrm{MB})$ and $67 \mathrm{~mL}(1 \mathrm{~L}-\mathrm{MB})(\mathrm{p}<0.001)$. In six patients, the mixed expired partial pressures of the six inert gases simultaneously collected from the two mixing boxes fell on the identity line. $V^{\prime} \mathrm{A} / Q^{\prime}$ distributions recovered using each of the two mixing boxes were equivalent. With the $1 \mathrm{~L}-\mathrm{MB}$, the effects of different positive end-expiratory pressure levels $\left(0,6\right.$ and $\left.12 \mathrm{cmH}_{2} \mathrm{O}\right)$ on $V \mathrm{c}$ and arterial carbon dioxide tension were negligible.

In conclusion, the new 1-L mixing box provides efficient gas mixing and substantially decreases the compression volume. It is, therefore, recommended when studies requiring mixed expired gas are performed in ventilated patients.

Eur Respir J 1998; 12: 1172-1176.
*Servei de Pneumologia i Al.lèrgia Respiratòria, Dept de Medicina, and +Unitat de Cures Intensives Quirúgica, Institut d'Investigacions Biomèdiques August Pi i Sunyer (IDIBAPS), Hospital Clínic, Universitat de Barcelona, Barcelona, Spain. \#Section of Physiology, University of California, San Diego, La Jolla, California, USA

Correspondence: J. Roca, Servei de Pneumologia, Hospital Clínic, Villarroel 170, Barcelona 08036, Spain. Fax: 34932275455

Keywords: Mechanical ventilation mixed expired gases

multiple inert gas elimination technique pulmonary gas exchange

ventilation-perfusion relationships

Received: April 151997

Accepted after revision July 81998

O. Díaz is an Associate Professor at the Universidad Pontificia Católica de Santiago de Chile, Chile. P.D. Wagner was a visiting Professor at the Universitat de Barcelona (PVI Program 1995).
The use of the multiple inert gas elimination technique (MIGET) [1-3] in clinical research has generated a large amount of information $[4,5]$ on the physiological abnormalities determining arterial oxygen $\left(\mathrm{Pa}_{\mathrm{a}} \mathrm{O}_{2}\right)$ and carbon dioxide $\left(\mathrm{Pa}_{\mathrm{a}} \mathrm{CO}_{2}\right)$ tensions in different conditions. The MIGET can be particularly useful in unravelling the factors governing pulmonary gas exchange in mechanically ventilated patients because, in addition to the determination of the corresponding ventilation-perfusion $\left(V^{\prime} \mathrm{A} / Q^{\prime}\right)$ distributions, it allows the impact of changes in extrapulmonary factors such as minute ventilation, cardiac output or $\mathrm{O}_{2}$ uptake on arterial blood gases to be quantified [6-10]. Simultaneous samples of arterial and mixed venous blood and mixed expired gases are obtained for inert gas measurements. Mixed expired gas sampling in the MIGET is usually obtained from a 10-L mixing box (10L-MB), heated above $37^{\circ} \mathrm{C}$, to obtain adequate gas mixing while avoiding loss of soluble gases, such as acetone and ether [5]. In mechanical ventilation, however, the compression volume $(V \mathrm{c})$, i.e. the volume diverted to the expiratory line of the ventilator circuit during a ventilatory cycle [11], generated by the $10 \mathrm{~L}-\mathrm{MB}$ placed in the expiratory side of the ventilator circuit, could be a major source of error in the estimation of the effective tidal volume $(V \mathrm{~T})$ delivered by the ventilator to the patient. This problem has markedly limited the com- parative analysis of the effects of different ventilatory modalities on pulmonary gas exchange $\left(V^{\prime} \mathrm{A} / Q^{\prime}\right.$ distributions).

It was hypothesized that by decreasing the compliance of the expiratory side of the ventilator circuit, by a volume reduction of the mixing expired gas, the $V_{c}$ could be minimized and the adequate expired gas mixing required by the MIGET could still be obtained. Accordingly, a new 1-L mixing box (1L-MB) was designed. The aims of the present study were to assess the impact of the $1 \mathrm{~L}-\mathrm{MB}$, as compared with the $10 \mathrm{~L}-\mathrm{MB}$, on the $V_{\mathrm{c}}$, in mechanically ventilated patients and using an in vitro model to simulate different ventilatory conditions. The efficacy of gas mixing was also assessed, along with the effects of the 1L-MB on arterial respiratory blood gases and the effects of different levels of positive end-expiratory pressure (PEEP; 0, 6 and $12 \mathrm{cmH}_{2} \mathrm{O}$ ) applied with the 1L-MB on both $V_{\mathrm{c}}$ and $\mathrm{Pa}, \mathrm{CO}_{2}$.

\section{Methods}

\section{The 1-L mixing box}

The new device consists of a hollow, stainless-steel cylinder of approximately $1 \mathrm{~L}$ internal volume, heated throughout its length using an electrical resistance to maintain a 
constant temperature between 40 and $50^{\circ} \mathrm{C}$. Ten discs placed as indicated in figure 1 are used to baffle the flow of gas through the cylinder, facilitating the gas mixing. The res-istance of the box is $0.31 \mathrm{cmH}_{2} \mathrm{O} \cdot \mathrm{L}^{-1} \cdot \mathrm{s}^{-1}$ at a flow of 1 $\mathrm{L} \cdot \mathrm{S}^{-1}$.

Effect of the 1-L and 10-L mixing boxes on the compression volume

A 1-L rubber bag, connected to a Siemens 900C Servo Ventilator (Siemens-Elema BA, Solna, Sweden), using regular tubing $(15 \mathrm{~mm}$ i.d., $150 \mathrm{~cm}$ length; Intertech Resources, Lincolnshire, IL, USA) was used as a surrogate lung. The characteristics of the circuit are illustrated in figure 2 . The $V \mathrm{~T}$ provided by the ventilator was measured with a pneumotachometer placed close to the inspiratory port of the ventilator and the effective $V \mathrm{~T}$ was obtained with a second pneumotachometer placed between the surrogate lung and the Y-piece of the ventilator circuit. Both pneumotachometers (21071B; Hewlett-Packard, Andover, MA, USA) were accurately calibrated using a rotameter and periodically checked during the study using a 3-L syringe. The peak airway pressure (P peak) applied by the ventilator was measured with a pressure transducer (Model 143PC-03D; Honeywell, Freeport, IL, USA) placed between the Y-piece of the ventilator circuit and the second pneu- motachometer. Flow and pressure were fed into a 80386 IBM-compatible computer via a 12-bit analogue-to-digital converter (Data Translation DT2801/ A; Data Translation, Marlboro, MA, USA) at a sampling rate of $100 \mathrm{~Hz}$ using Anadat software (RHT-InfoData, Montreal, Canada). Tidal volume, respiratory frequency $(f \mathrm{R})$, inspiratory time $(t \mathrm{I})$ and total cycle duration ( $t$ tot $)$ were calculated over 30-s periods of continuous recording for each flow signal. $V_{\mathrm{c}}$ was calculated as the difference between the $V \mathrm{~T}$ delivered by the ventilator and the $V \mathrm{~T}$ that effectively reached the surrogate lung. Measurements, at an inspiratory oxygen fraction $\left(F \mathrm{I}, \mathrm{O}_{2}\right)$ of 0.21 without applying PEEP, were taken using the in vitro model (fig. 2) in three different conditions: 1 ) with the ventilator circuit

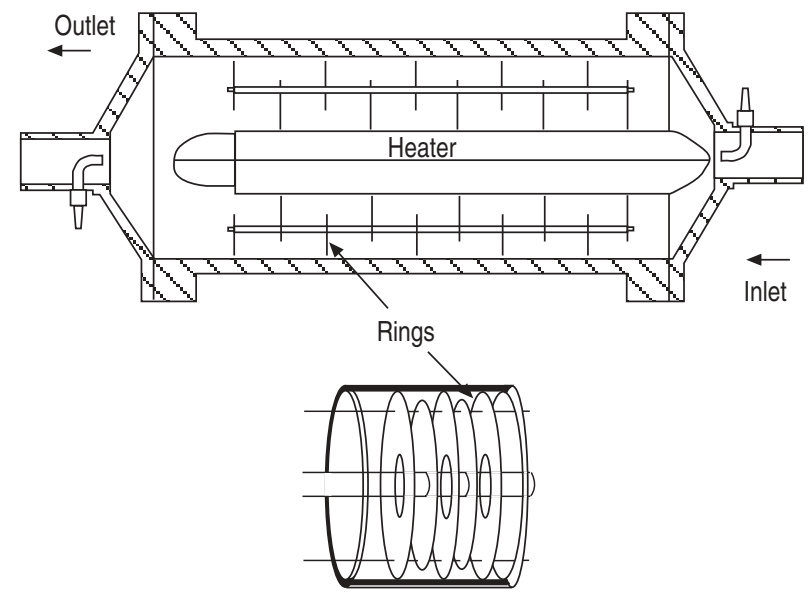

Fig. 1. - Schematic representation of the newly designed 1-L mixing box, a hollow stainless-steel cylinder heated throughout its length using an electrical resistance, with 10 discs placed transversely to baffle the flow of gas through the cylinder, facilitating the gas mixing. The mixed expired gas samples are obtained at the outlet through a collateral connection.

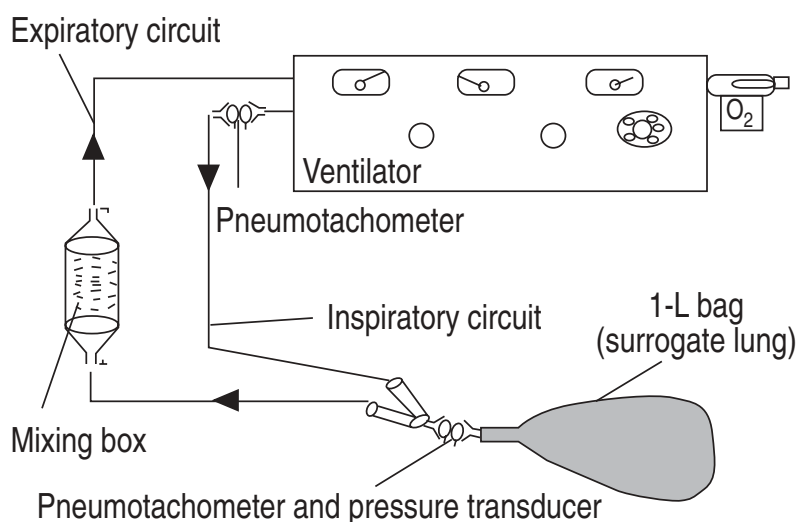

Fig. 2. - Schematic representation of the circuit used to assess the effect of the 1-L and 10-L mixing boxes on the compression volume. A $1-\mathrm{L}$ bag was used as a surrogate lung. Two pneumotachometers, placed close to the inspiratory port of the ventilator and between the surrogate lung and the Y-piece of the ventilator, were used to measure tidal volume $(V \mathrm{~T})$ provided by the ventilator and effective $V \mathrm{~T}$, respectively.

alone; 2) with the 10L-MB inserted in the expiratory limb of the ventilator circuit; and 3) with the $1 \mathrm{~L}-\mathrm{MB}$ in the circuit. Because $V_{\mathrm{c}}$ varies according to end-inspiratory airway pressure [12], $V_{c}$ was measured at step increases of $P$ peak from $10-50 \mathrm{cmH}_{2} \mathrm{O}$ in each condition. The step changes in $P$ peak were generated by increasing the volume delivered by the ventilator, with no modifications in either the $f \mathrm{R}$ or in the $t \mathrm{t} / t$ tot ratio. Values of $V_{\mathrm{c}}$ obtained with no mixing box in the ventilator circuit, thus representing the $V_{\mathrm{c}}$ of the circuit alone, were then subtracted from those obtained at the same levels of $P$ peak with the 10L-MB or the $1 \mathrm{~L}-\mathrm{MB}$ attached to the circuit.

\section{Effect of the 1-L mixing box on arterial blood gases.}

The effects of the 1L-MB on $V_{c}$ and on arterial blood gases were assessed in six consecutive patients admitted to the intensive care unit and requiring mechanical ventilation (age $70 \pm 13$ yrs, $F \mathrm{I}_{2} \mathrm{O}_{2} 0.29 \pm 0.02$ ). Two patients had pneumonia, two had chronic airflow limitation, one had acute lung injury and one had a brain haemorrhage. Patients were sedated and paralysed and the baseline ventilatory setting (without PEEP) was kept constant throughout the study. Measurements were performed, in random order, before and $30 \mathrm{~min}$ after assembling the $1 \mathrm{~L}-\mathrm{MB}$ in random order. $P \mathrm{a}, \mathrm{O}_{2}, \mathrm{~Pa}_{\mathrm{a}} \mathrm{CO}_{2}$, and $\mathrm{pH}$ were measured by a standard electrode technique (IL-1306; Instrumentation Laboratories, Milan, Italy). Peak airway pressures and $V_{\mathrm{c}}$ were obtained following the procedure described above. In three additional patients with acute lung injury (age $61 \pm 15 \mathrm{yrs}, F \mathrm{I}, \mathrm{O}_{2}$ $0.47 \pm 0.12)$, the effects of PEEP $\left(0,6\right.$ and $\left.12 \mathrm{cmH}_{2} \mathrm{O}\right)$ using the $1 \mathrm{~L}-\mathrm{MB}$ on $\mathrm{Pa}_{\mathrm{a}} \mathrm{CO}_{2}$ and $V_{\mathrm{c}}$ were examined.

\section{Assessment of mixed expired gas sampling}

A further six patients (age $57 \pm 6$ yrs, $F \mathrm{I}, \mathrm{O}_{2} 0.40 \pm 0.12$ ) with respiratory failure requiring volume-controlled mechanical ventilation (three had chronic airflow limitation, one had thoracic trauma, one had acute lung injury and one had a drug overdose) were studied using two different levels of 
effective $V \mathrm{~T}, 400$ and $800 \mathrm{~mL}$, obtained by changing the respiratory rate to keep the effective minute ventilation unchanged. This protocol compared the mixed expired partial pressures of the six inert gases of the MIGET simultaneously collected from the 10L-MB and the 1L-MB. The two mixing boxes were placed in series in the expiratory limb of the ventilator circuit with the 1L-MB proximal and the 10L-MB distal to the patient. The two ventilatory settings (VT 400 and $800 \mathrm{~mL}$ ) were applied to each patient in a random order. Patients were also sedated and paralysed during this protocol. No PEEP was applied.

The MIGET is based on the simultaneous elimination of trace concentrations of six inert gases (sulphur hexafluoride (SF6), ethane, cyclopropane, halothane, ether and acetone) infused, in a saline solution, into a peripheral vein of the forearm [1-5]. The general features of the setup of the MIGET in the authors' laboratory have been reported elsewhere $[6,7]$. In brief, after approximately $45 \mathrm{~min}$ of infusion of the solution of the six inert gases at a rate of 3 $\mathrm{mL} \cdot \mathrm{min}^{-1}$, arterial blood $(8 \mathrm{~mL})$ and mixed expired gas samples from each of the two mixing boxes were simultaneously obtained in duplicate after steady-state conditions were ensured. The arterial blood and each set of mixed expired gas measurements (1L-MB and 10L-MB) were treated separately to obtain the corresponding $V^{\prime} \mathrm{A} / Q^{\prime}$ distributions, with the average of duplicate measurements used in the analysis. The same value for cardiac output was assumed for the calculation of the $V^{\prime} \mathrm{A} / Q^{\prime}$ distributions.

\section{Safety procedures}

The electrocardiogram and systemic blood pressure were monitored continuously (System Sirecust 404 and 455-1; Siemens Aktiengesellschaft, Erlangen, Germany). Arterial $\mathrm{O}_{2}$ saturation was monitored during the study by pulse oximetry (504 Pulse Oximeter; Criticare Systems, Waukesha, WI, USA) and maintained $>96 \%$. The end-inspiratory plateau pressure with the higher tidal volume $(V \mathrm{~T} 800 \mathrm{~mL})$ remained $<30 \mathrm{cmH}_{2} \mathrm{O}$. The study protocol was approved by the Ethical Research Committee of the Hospital Clinic. Informed consent was given by all the patients participating in the study, or by their next of kin.

\section{Data analysis}

Results are expressed as mean \pm SD. Plots of $V_{c}$ versus $P$ peak were used to represent the mechanical behaviour of the two mixing boxes. The volume-pressure relationships were subsequently explored by standard regression analysis. Concordance between pairs of mixed expired partial pressures obtained from the two mixing boxes was assessed for the six inert gases by calculating the intraclass correlation coefficient based on the one-way random effects model analysis of variance (ANOVA) [13]. The correlation coefficient obtained is only equal to 1 when all the pairs of values fall on a straight line through the origin with slope unity. In addition, the Student's paired t-test was used to determine whether differences between those pairs deviated significantly from zero. Values of mean (first moment) and standard deviation (second moment) of both ventilation and perfusion distributions $\left(V^{\prime} \mathrm{A} / Q^{\prime}\right)$ recovered from each mixing box were also compared. Statistical significance was set at ð0.05.

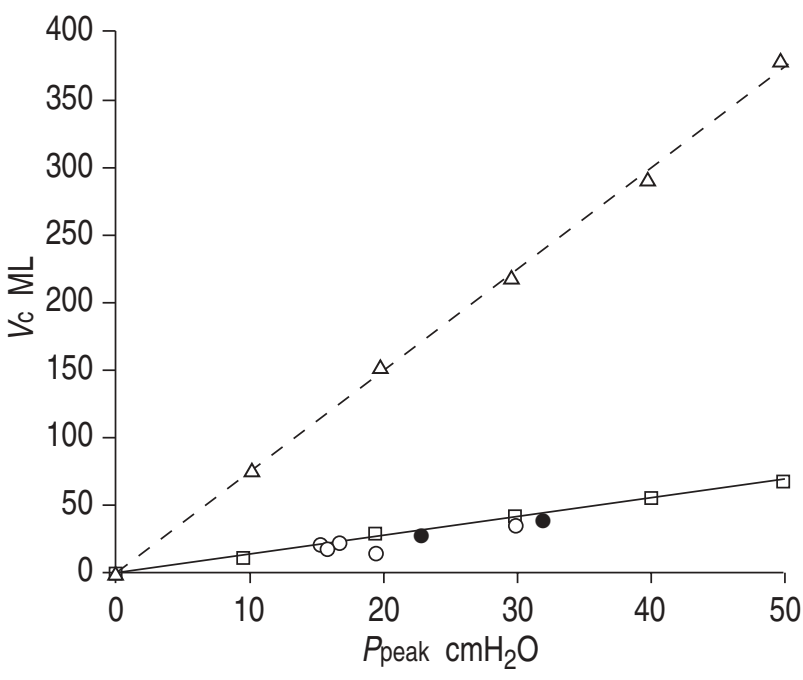

Fig. 3. - Relationship between peak airway pressures ( $P$ peak $)$ applied by the ventilator and the compression volume $\left(V_{\mathrm{c}}\right)$ for both the 1-L mixing box $(1 \mathrm{~L}-\mathrm{MB} ; \square)$ and the $10-\mathrm{L}$ mixing box $(10 \mathrm{~L}-\mathrm{MB}$; ý). Regression lines fitting data points obtained with the surrogate $(1 \mathrm{~L}-\mathrm{MB} ;-; 10 \mathrm{~L}-$ MB, - - -) are shown. Results obtained using the 1L-MB in five mechanically ventilated patients are also plotted $(\mathrm{O})$ and a single patient $(\bullet)$ at two levels of tidal volume (400 and $800 \mathrm{~mL}$ ).

\section{Results}

\section{Assessment of the compression volume}

Figure 3 shows the relationships between $P$ peak and the corresponding values of $V_{\mathrm{c}}$ showed by the $1 \mathrm{~L}-\mathrm{MB}$ and the 10L-MB. In both conditions, $V \mathrm{c}$ was linearly related to $P$ peak (r=0.99 in both cases). The relationships between $P$ peak $\left(\mathrm{cmH}_{2} \mathrm{O}\right)$ and $V_{\mathrm{c}}(\mathrm{mL})$ were defined by $V_{\mathrm{c}}=7.4 \mathrm{~mL} \cdot \mathrm{cmH}_{2} \mathrm{O}$. $P$ peak $^{-1}$ and $V \mathrm{c}=1.4 \mathrm{~mL} \cdot \mathrm{cmH}_{2} \mathrm{O} \cdot P$ peak $^{-1}$ in the $10 \mathrm{~L}-\mathrm{MB}$ and the $1 \mathrm{~L}-\mathrm{MB}$, respectively.

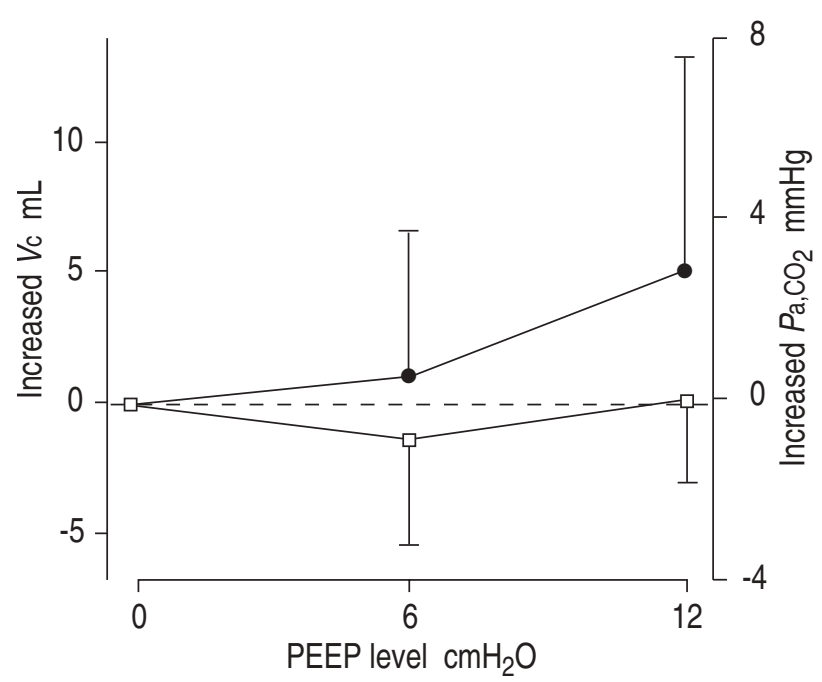

Fig. 4. - Effects of positive end-expiratory pressure (PEEP) on compression volume $\left(V_{\mathrm{c}}\right)$ and arterial carbon dioxide tension $\left(\mathrm{P}_{\mathrm{a}}, \mathrm{CO}_{2}\right)$ using the 1-L mixing box. Results are expressed as mean \pm SEM of the differences in $V_{\mathrm{c}}(\bullet)$ and in $\mathrm{Pa}_{\mathrm{a}} \mathrm{CO}_{2}(\square)$ at two levels of PEEP (6 and 12 $\left.\mathrm{cmH}_{2} \mathrm{O}\right)$, compared with baseline $\left(0 \mathrm{cmH}_{2} \mathrm{O}\right) .(1 \mathrm{mmHg}=0.133 \mathrm{kPa}$. $)$ 
Effects of the 1-L mixing box on arterial blood gases

The $V_{\mathrm{c}}$ developed by the $1 \mathrm{~L}-\mathrm{MB}$ in the six patients fell on the regression line obtained with the surrogate lung (fig. 3). The effect of the 1L-MB on arterial blood gases was modest, accounting for a mild, clinically irrelevant increase in $\mathrm{Pa}, \mathrm{CO}_{2}$ (from $37.4 \pm 7.6$ to $39.7 \pm 7.7 \mathrm{mmHg}$ ) ( $\mathrm{p}<$ $0.005)$, with no changes in arterial blood $\mathrm{pH}(7.45 \pm 0.07$ to $7.44 \pm 0.07$ ) or the $P \mathrm{a}, \mathrm{O}_{2} / F \mathrm{I}_{1} \mathrm{O}_{2}$ ratio (from $337 \pm 55$ to $338 \pm$ 78 ). No induced PEEP was observed when the 1L-MB was placed in the ventilator circuit. Moreover, the use of different levels of PEEP $\left(0,6\right.$ and $\left.12 \mathrm{cmH}_{2} \mathrm{O}\right)$ did not show any substantial effects on either $V_{c}$ or $P_{a}, \mathrm{CO}_{2}$, (fig. 4).

\section{Assessment of the mixed expired gas sampling}

Figure 5 indicates that similar values of mixed expired partial pressures of the six inert gases were obtained from a) 65

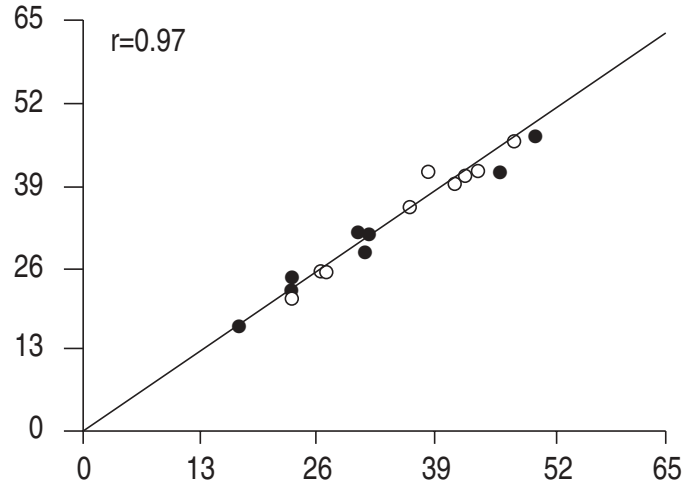

c)

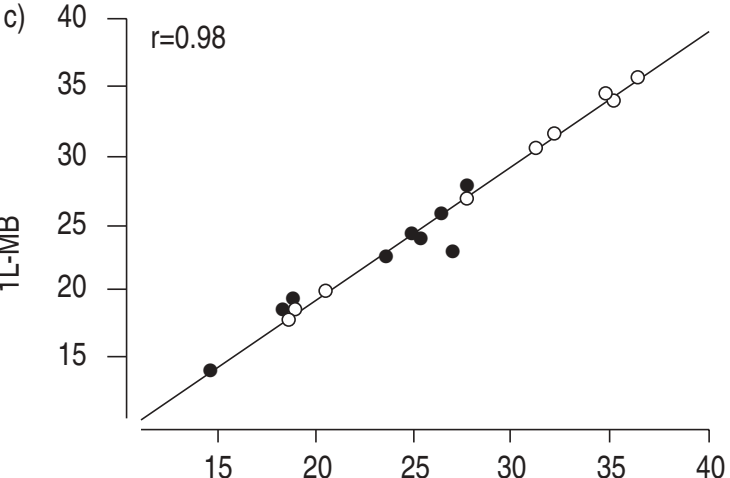

e)

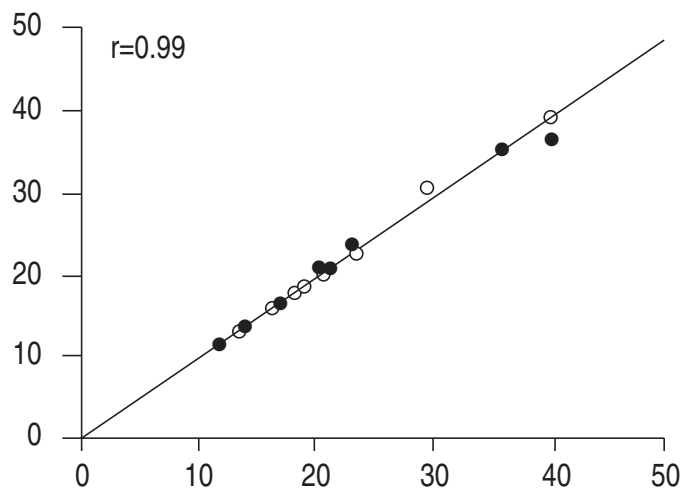

the two mixing boxes, irrespective of the inert gas or the value of $V \mathrm{~T}$. The concordance in gas mixing between the two boxes is also demonstrated by the high intraclass correlation coefficients, ranging $0.96-0.99$ for the six inert gases. Mean differences between these pairs of values were not significantly different from zero. Accordingly, no differences in the first and second moments of the recovered $V^{\prime} \mathrm{A} / Q^{\prime}$ distributions were observed between the two mixing boxes.

\section{Discussion}

The main findings of this study were that a large increase in $V_{\mathrm{c}}$ was provoked by the 10L-MB, but was not present with the newly designed $1 \mathrm{~L}-\mathrm{MB}$, and that the $1 \mathrm{~L}-$ MB showed similar efficacy to the 10L-MB on expired
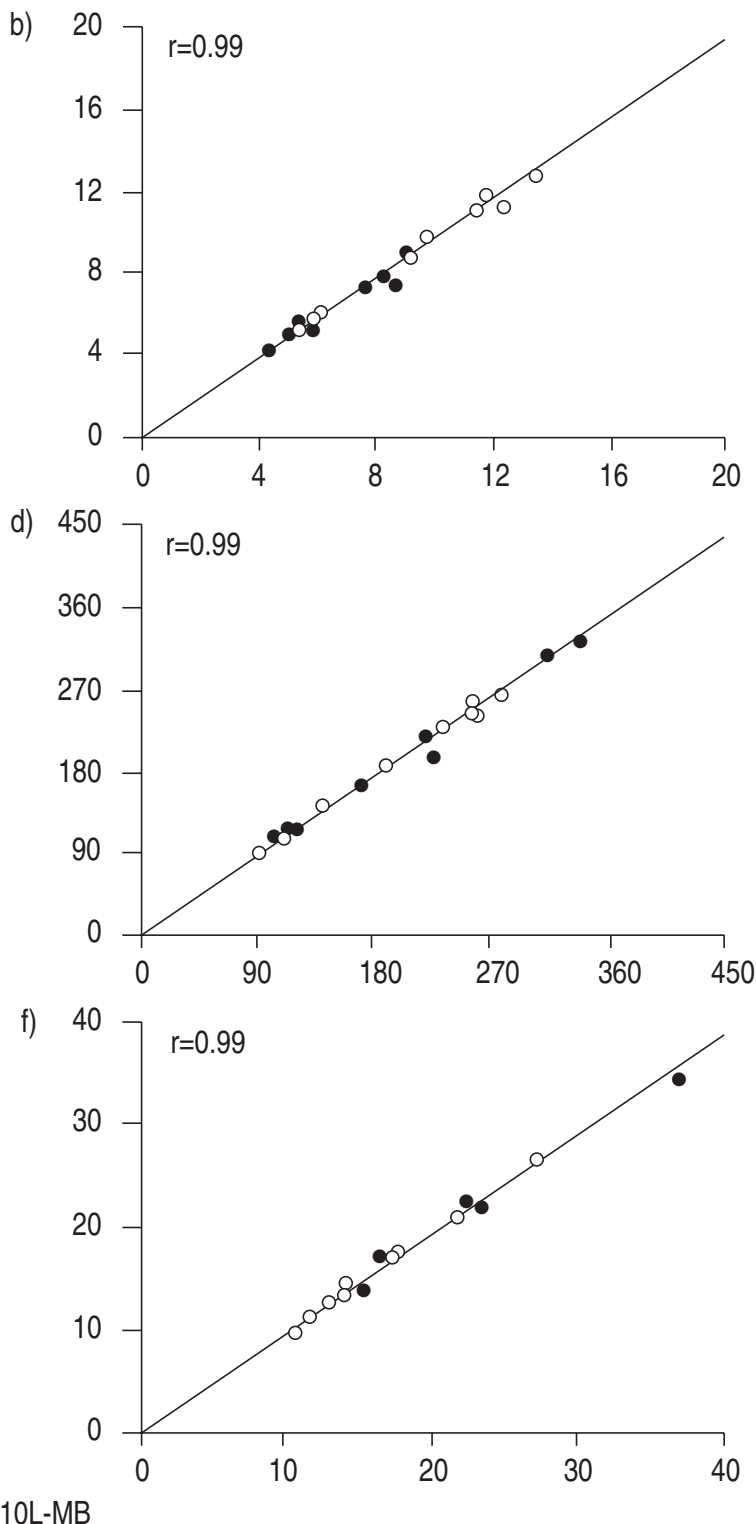

Fig. 5. - Identity plots of expired partial pressures of the six inert gases used in the multiple inert gas elimination technique, sampled simultaneously from the 10-L mixing box (10L-MB) and from the 1-L mixing box (1L-MB) in mechanically ventilated patients. a) Sulphur hexafluoride; b) ethane; c) cyclopropane; d) halothane; e) ether; f) acetone. Measurements were performed at two levels of tidal volume: $400 \mathrm{~mL}(\mathrm{O})$ and $800 \mathrm{~mL}(\bullet)$. Partial pressures of inert gases are dimensionless. 
gas mixing. The present study validates the $1 \mathrm{~L}-\mathrm{MB}$ as an appropriate tool to explore the effects of different ventilatory modalities on pulmonary gas exchange $\left(V^{\prime} \mathrm{A} / Q^{\prime}\right.$ distributions), overcoming the previous drawbacks of the MIGET application in this field.

The first conclusion was not unexpected, owing to the major differences in internal volume between the two boxes. In consequence, at all levels of Ppeak imposed by the ventilator, $V \mathrm{c}$ was almost six-times higher with the 10LMB than with the $1 \mathrm{~L}-\mathrm{MB}(\mathrm{p}<0.0001)$. This is clearly shown in figure 3 , where at a $P$ peak of $10 \mathrm{cmH}_{2} \mathrm{O}$, the $V_{\mathrm{c}}$ was 76 $\mathrm{mL}(10 \mathrm{~L}-\mathrm{MB})$ and $12 \mathrm{~mL}(1 \mathrm{~L}-\mathrm{MB})$, and at a $P$ peak of 50 $\mathrm{cmH}_{2} \mathrm{O}, V_{\mathrm{c}}$ increased to $377 \mathrm{~mL}$ and $67 \mathrm{~mL}$, respectively. It is of note that the values of $V_{c}$ reported here only represent those produced by the mixing boxes. To obtain the total $V_{\mathrm{c}}$ of a particular ventilator tubing system, the compression volume of the tubing would need to be added to the reported values. In view of these findings, it seems clear that the use of the 10L-MB during mechanical ventilation can lead to significant overestimation of $V \mathrm{~T}$, with the magnitude related to the pressure applied by the ventilator. The effect of the 10L-MB on $V_{c}$ could be particularly troublesome whenever the mechanical characteristics of the system change over time, as may be the case when different modes of mechanical ventilation are applied. Then, $V$ T can change in an uncontrolled manner, possibly inducing alveolar hypoventilation, provoking a clinical as well as a research problem, unless the effective $V \mathrm{~T}$ delivered to the patient is controlled as in the present study or no attempts are made to match for $V \mathrm{~T}[6,7]$.

In the present study, the 10L-MB was used as a reference only because it is the older and more common way of obtaining mixed expired samples in the MIGET during spontaneous breathing. Two alternative methods to prevent $V$ c-related problems can be used in mechanical ventilation. Firstly, placing the mixing box after the expiratory valve, at the ventilator gas outlet, would be an adequate solution to prevent $V_{\mathrm{c}}$ secondary to the mixing box, but the potential loss of soluble gases such as acetone and ether throughout the ventilator circuit could be a source of methodological errors unless a specific ventilator designed to avoid these problems by heating all of the circuit is available. Although BeyDon et al. [10] did not find any major problems in placing the mixing box after the expiratory valve, potential errors with this approach are always ventilator specific. Secondly, the use of a pneumatic valve as a Y-piece close to the patient's trachea, separating the inspiratory and the expiratory circuit, is a potentially adequate method for obtaining noncontaminated expired gas, but it did not prove practical in the present study, since there was major interference with the ventilator.

The reduction in size of the $1 \mathrm{~L}-\mathrm{MB}$ to limit $V_{\mathrm{c}}$ did not affect the accuracy of mixed expired gas sampling in the MIGET. This accuracy was reflected by the close agreement in the expired partial pressures of the six inert gases between the 1L-MB and the 10L-MB. Moreover, the potential of the 1L-MB to increase functional residual capacity and PEEP is negligible owing to the low expiratory resistance $\left(0.31 \mathrm{cmH}_{2} \mathrm{O} \cdot \mathrm{L}^{-1} \cdot \mathrm{s}^{-1}\right.$ at a flow of $\left.1 \mathrm{~L} \cdot \mathrm{min}^{-1}\right)$ generated by the box, which is similar to that generated by the $10 \mathrm{~L}-\mathrm{MB}$, as observed in the present study. In addition, no intrinsic PEEP associated with any of the mixing boxes was shown.
In summary, this study demonstrates that mixed expired gas sampling in mechanically ventilated patients can be conducted using a mixing box of considerably smaller size (1 $\mathrm{L})$ than the $10-\mathrm{L}$ mixing box. The newly designed 1-L mixing box not only proved to be as efficient as the 10-L mixing box but also substantially decreased the compression volume. Moreover, it can be used independently of the type of ventilator. Accordingly, the 1-L mixing box proposed in this study is recommended to assess adequately the effects of mechanical ventilation on gas exchange, especially when different ventilatory conditions are studied $[14,15]$.

Acknowledgements: The authors are indebted to F. Burgos for his technical assistance and to J. Cardús for skilful application of the inert gas technique.

\section{References}

1. Wagner PD, Naumann PF, Laravuso RB. Simultaneous measurement of eight foreign gases in blood by gas chromatography. J Appl Physiol 1974; 36: 600-605.

2. Wagner PD, Saltzman HA, West JB. Measurements of continuous distributions of ventilation-perfusion ratios: theory. J Appl Physiol 1974; 36: 588-599.

3. Evans JW, Wagner PD. Limits of $V^{\prime} \mathrm{A} / Q^{\prime}$ distributions from analysis of experimental inert gas data elimination. J Appl Physiol 1977; 42: 889-898.

4. Wagner PD, Rodriguez-Roisin R. Clinical advances in pulmonary gas exchange. Am Rev Respir Dis 1991; 143: 883-888

5. Roca J, Wagner PD. Principles and information content of the multiple inert gas elimination technique. Thorax 1994; 49: 815-824.

6. Torres A, Reyes A, Roca J, Wagner PD, Rodriguez-Roisin R. Ventilation-perfusion mismatching in chronic obstructive pulmonary disease during ventilator weaning. Am Rev Respir Dis 1989; 140: 1246-1250.

7. Rodriguez-Roisin R, Ballester E, Roca J, Torres A, Wagner PD. Mechanisms of hypoxemia in patients with status asthmaticus requiring mechanical ventilation. Am Rev Respir Dis 1989; 139: 732-739.

8. Valentine DD, Hammond MD, Downs JB, Sears NJ, Sims WR. Distribution of ventilation and perfusion with different modes of mechanical ventilation. Am Rev Respir Dis 1991; 143: 1262-1266.

9. Santak B, Radermacher P, Sandman W, Falke KJ. Influence of SIMV plus inspiratory pressure support on $V^{\prime} \mathrm{A} / Q^{\prime}$ distributions during postoperative weaning. Intensive Care Med 1991; 17: 136-140.

10. Beydon L, Cinotti L, Rekik N, et al. Changes in the distribution of ventilation and perfusion associated with separation from mechanical ventilation in patients with obstructive pulmonary disease. Anesthesiology 1991; 75: 730-738.

11. Bartel LP, Bazik JR, Powner DJ. Compression volume during mechanical ventilation: comparison of ventilators and tubing circuits. Crit Care Med 1985; 13: 851-854.

12. Tobin MJ, Van de Graaff WB. Monitoring of lung mechanics and work of breathing. In: Tobin MJ, ed. Principles and Practice of Mechanical Ventilation, 1st Edn. New York, McGraw-Hill, 1994; pp. 967-1003.

13. Armitage P, Berry G. Statistical Methods in Medical Research, 3th Edn. London, Blackwell Scientific Publications, 1994; pp. 273-276.

14. Diaz O, Iglesia R, Ferrer M, et al. Effects of noninvasive ventilation on pulmonary gas exchange and hemodynamics during acute hypercapnic exacerbations of chronic obstructive pulmonary disease. Am J Respir Crit Care Med 1997; 156: 1840-1845.

15. Zavala E, Ferrer M, Polese G, et al. Effect of inverse I:E ratio ventilation on pulmonary gas exchange in acute respiratory distress syndrome. Anesthesiology 1998; 88: 35-42. 